\title{
Transcriptomic Landscape of Medicinal Dendrobium Reveals Genes Associated With the Biosynthesis of Bioactive Components
}

OPEN ACCESS

Edited by:

Guodong Wang,

Institute of Genetics

and Developmental Biology, Chinese

Academy of Sciences, China

Reviewed by:

Sheng Lin,

Chinese Academy of Medical

Sciences and Peking Union Medical

College, China

Sibongile Mafu,

University of Massachusetts Amherst,

United States

*Correspondence:

Zhicai Wang

wzcxjnu.5525@163.com

Meina Wang

wangmn@cnocc.cn

Specialty section:

This article was submitted to

Plant Metabolism

and Chemodiversity,

a section of the journal

Frontiers in Plant Science

Received: 20 December 2019 Accepted: 18 March 2020

Published: 28 April 2020

Citation:

Wang Z, Zhao M, Cui H, Li J and Wang M (2020) Transcriptomic Landscape of Medicinal Dendrobium

Reveals Genes Associated With the Biosynthesis of Bioactive

Components.

Front. Plant Sci. 11:391. doi: 10.3389/fp/s.2020.00391

\author{
Zhicai Wang ${ }^{1,3 *}$, Meili Zhao ${ }^{1}$, Hongqiu Cui ${ }^{1}$, Jian Li ${ }^{1}$ and Meina Wang ${ }^{1,2 *}$ \\ ${ }^{1}$ Key Laboratory of National Forestry and Grassland Administration for Orchid Conservation and Utilization, Shenzhen, \\ China, ${ }^{2}$ Shenzhen Key Laboratory for Orchid Conservation and Utilization, The National Orchid Conservation Center \\ of China and The Orchid Conservation \& Research Center of Shenzhen, Shenzhen, China, ${ }^{3}$ Key Laboratory of Medical \\ Reprogramming Technology, Shenzhen Second People's Hospital, The First Affiliated Hospital of Shenzhen University, \\ Shenzhen, China
}

Many plants of Dendrobium genus are precious traditional herbs with high commercial value and excellent medicinal effects. They are perennial aerophytes or epiphytes of terrestrial orchids growing on cliffs and tree trunks covered with mosses in forests throughout the tropical and subtropical Asia and eastern Australia. The stem contains a variety of bioactive components, including polysaccharides and alkaloids, with strong antioxidant, neuroprotective, and immunomodulatory effects. Great attention has been drawn to the Dendrobium genus regarding its medicinal effectiveness, and the related researches have been accumulating rapidly in recent years. The bioactive components are mainly the intermediates or final products produced in specialized metabolite biosynthesis. Thus far, the activity, molecular structure, and composition of major medicinal ingredients have been partially elucidated, and the sequencing of several transcriptomes has been starting to shed new light on the biosynthesis regulation mechanism. This paper reviewed the advances of researches concerning the biosynthetic pathways of medicinal specialized metabolites from Dendrobium, especially the large number of related genes, with the hope of further promoting the development and utilization of those components and correspondingly protecting the Dendrobium resources in more effective ways.

Keywords: Dendrobium, specialized metabolites, medicinal components, biosynthesis, transcriptome

\section{INTRODUCTION}

Dendrobium, one of the largest genera in the Orchidaceae family with more than 1500 species worldwide, is mainly distributed throughout India, southern Asia, Japan, Australia, and some Pacific islands (Wang et al., 2009; Pridgeon et al., 2014). Several Dendrobium species including D. nobile, D. officinale (D. catenatum), D. huoshanense, and D. chrysanthum, are highly prized folk medicines (Table 1) in many Asian countries for hundreds of years with special pharmacological effects on inflammation, gastritis, diabetes, cancer, and aging (Ng et al., 2012; Song et al., 2012). 
TABLE 1 | The effective components and their bioactivities in medicinal Dendrobium.

\begin{tabular}{|c|c|c|c|}
\hline $\begin{array}{l}\text { Bioactive } \\
\text { constituents }\end{array}$ & Species names & Bioactivity & References \\
\hline Alkaloids & D. nobile & Neuroprotective activity & Li et al., 2011 \\
\hline Bisbenzyls & D. nobile & Antifungal activities & Zhou et al., 2016 \\
\hline Dendroflorin & D. nobile & Antisenescence & Jin et al., 2008 \\
\hline Flavonoids & D. officinale & Antioxidant, antitoxicity & Prochazkova et al., 2011; Wang et al., 2017 \\
\hline $\begin{array}{l}\text { Glucosyloxycinnamic } \\
\text { acid derivatives }\end{array}$ & D. aurantiacum & Antioxidant & Yang et al., 2004 \\
\hline Lectin & D. findleyanum, D. officinale & Hemagglutinating, antifungal & Sattayasai et al., 2009 \\
\hline Moscatilin & D. loddigesii & Suppresses tumor angiogenesis and growth & Tsai et al., 2010 \\
\hline Polysaccharides & D. huoshanense, D. officinale & $\begin{array}{l}\text { Immunomodulatory, hepatoprotective, and } \\
\text { antioxidant activities }\end{array}$ & Liu et al., 2011; Wu et al., 2011; Pan et al., 2012 \\
\hline Phenanthrenes & D. loddigesii & Antioxidant & Ito et al., 2010 \\
\hline Trigonopol A & D. trigonopus & Inhibits platelet aggregation & Hu et al., 2008 \\
\hline
\end{tabular}

The major active ingredients include polysaccharides (Luo et al., 2009), alkaloids (Wang Q. et al., 2010), bibenzyls (Yang et al., 2006), flavonoids (Lei et al., 2018), amino acids, and several trace mineral elements (Guo et al., 2013). Due to over-exploitation and deterioration of natural habitats, most of the wild Dendrobium species have been increasingly endangered.

Transcriptomic analysis is a powerful tool for exploring specialized metabolite biosynthetic genes and their expression patterns, which can be used to determine the synthesis and metabolic pathway. Recently, a large number of putative genes involved in the biosynthesis of polysaccharides (Shen et al., 2017), alkaloids (Li Q. et al., 2017), and flavonoids (Lei et al., 2018) have been identified in Dendrobium through transcriptome sequencing (Table 2 and Figure 1). For instance, in D. officinale, the first transcription sequencing data that revealed the genes associated with alkaloid biosynthesis were published in 2013 (Guo et al., 2013). In 2016, a transcriptome study focusing on the regulatory maps in response to cold acclimation, polysaccharide synthesis, and gene expression profiling of the protocorm has been conducted (Zhang J. et al., 2016). In that same year, the genome of $D$. catenatum was sequenced and the polysaccharide synthetic pathway was analyzed (Zhang G.Q. et al., 2016). Later, in 2017 (Shen et al., 2017), unigenes associated with fructose and mannose metabolism and the putative alkaloid biosynthetic pathway were identified, while in D. nobile, transcriptome analysis was carried out to reveal genes related to the biosynthesis of dendrobine through the mevalonate (MVA) pathway (Li Q. et al., 2017). Furthermore, genes in the polysaccharide synthetic pathway, including cellulose synthase-like A 6 (DoCSLA6), UDP galacturonate 4epimerase (DoUGE), UDP-glucose pyrophosphorylase (DoUGP), and GDP-mannose pyrophosphorylase 1 (DoGMP1) have been cloned and functionally characterized in D. officinale (Fan et al., 2016). The purpose of this review is to summarize the advances in transcriptome-related studies, with an emphasis on functional characterization of regulatory genes related to some of the major active ingredient biosynthesis in recent years and to provide useful insights into the further dissection of biosynthesis regulation mechanism in Dendrobium.

\section{DENDROBIUM ALKALOID BIOSYNTHESIS}

\section{Dendrobium Alkaloid}

The major medicinal constituents of Dendrobium include alkaloids, flavonoids, polysaccharides, polyphenols, etc. ( $\mathrm{Lu}$ et al., 2014). Among these compounds, alkaloids are the most important medicinal components and the first category extracted and characterized from Dendrobium plants. Thus far, five types of structurally confirmed alkaloids including sesquiterpene alkaloids, imidazole alkaloids, phthalide alkaloids, pyrrolidine

TABLE 2 | Transcriptome sequencing of medicinal Dendrobium revealing genes related to specialized metabolites production.

\begin{tabular}{|c|c|c|c|c|}
\hline $\begin{array}{l}\text { Dendrobium } \\
\text { species }\end{array}$ & $\begin{array}{l}\text { Sequencing } \\
\text { time }\end{array}$ & $\begin{array}{l}\text { Sequencing } \\
\text { country }\end{array}$ & $\begin{array}{l}\text { Sequencing } \\
\text { platforms }\end{array}$ & References \\
\hline D. officinale & 2013 & China & $\begin{array}{l}\text { Roche } 454 \text { GS } \\
\text { FLX Titanium } \\
\text { platform }\end{array}$ & $\begin{array}{l}\text { Guo et al., } \\
2013\end{array}$ \\
\hline D. officinale & 2015 & China & $\begin{array}{l}\text { Illumina HiSeq } \\
2000\end{array}$ & $\begin{array}{l}\text { He et al., } \\
2015\end{array}$ \\
\hline D. officinale & 2016 & China & $\begin{array}{l}\text { Illumina HiSeq } \\
2000\end{array}$ & $\begin{array}{l}\text { Zhang J. } \\
\text { et al., } 2016\end{array}$ \\
\hline D. officinale & 2016 & China & $\begin{array}{l}\text { Illumina HiSeq } \\
2000\end{array}$ & $\begin{array}{l}\text { An et al., } \\
2016\end{array}$ \\
\hline D. officinale & 2017 & China & $\begin{array}{l}\text { Illumina HiSeq } \\
2500\end{array}$ & $\begin{array}{l}\text { Shen et al., } \\
2017\end{array}$ \\
\hline D. nobile & 2017 & China & $\begin{array}{l}\text { Illumina HiSeq } \\
4000\end{array}$ & $\begin{array}{l}\text { Li Q. et al., } \\
2017\end{array}$ \\
\hline D. officinale & 2017 & China & $\begin{array}{l}\text { Illumina HiSeq } \\
1500\end{array}$ & $\begin{array}{l}\text { He et al., } \\
2017 b\end{array}$ \\
\hline D. huoshanense & 2018 & China & $\begin{array}{l}\text { Illumina HiSeq } \\
2500\end{array}$ & $\begin{array}{l}\text { Yuan et al., } \\
2018\end{array}$ \\
\hline D. catenatum & 2018 & China & $\begin{array}{l}\text { Illumina HiSeq } \\
4000\end{array}$ & $\begin{array}{l}\text { Lei et al., } \\
2018\end{array}$ \\
\hline D. officinale & 2019 & China & $\begin{array}{l}\text { Illumina HiSeq } \\
4000\end{array}$ & $\begin{array}{l}\text { Chen et al., } \\
2019\end{array}$ \\
\hline $\begin{array}{l}\text { D. huoshanense, } \\
\text { D. officinale, and } \\
\text { D. moniliforme }\end{array}$ & 2020 & China & Not available & $\begin{array}{l}\text { Yuan et al., } \\
2020\end{array}$ \\
\hline
\end{tabular}


2016 Transcriptome analysis identified candidate genes involved in polysaccharide biosynthesis in $D$. officinale (Zhang J. et al., 2016)
December 2018 D. huoshanense transcriptome unveiled putative genes associated with polysaccharide and alkaloid synthesis (Yuan et al., 2018)

\begin{abstract}
2013 The first transcription sequence data was published in $D$. officinate revealing 25 genes that involved in alkaloid backbone biosynthesis (Guo et al., 2013)
\end{abstract}

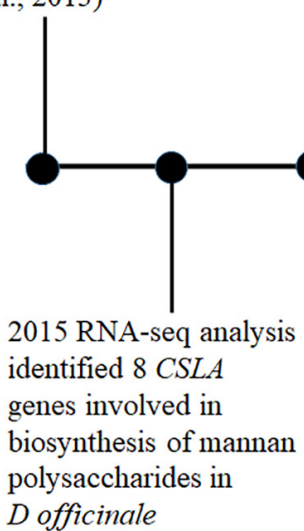

(He et al., 2015)
March 2017 Transcriptome analysis of $D$. nobile infected with fungus $M F 23$ revealed 30 genes related to dendrobine biosynthesis (Li Q. et al., 2017)

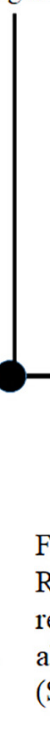

\author{
Feb
}

February 2017 Organ-specific RNA-seq revealed genes related to polysaccharide and alkaloid synthesis in $D$. officinale (Shen et al., 2017)

April 2018 Transcriptome analysis

2020 Transcriptome and gene co-expression network analysis of three Dendrobium species revealed active ingredients-related genes and pathways (Yuan et al., 2020) revealed genes associated with flavonoid biosynthesis in D. catenatum from different locations (Lei et al., 2018)

FIGURE 1 | Timeline of transcriptome studies probing the biosynthesis pathways for active ingredients in medicinal Dendrobium. Notable studies were included and the key references for each study were given.

alkaloids, and indolizidine alkaloids have been clarified $(\mathrm{Ng}$ et al., 2012). Despite the complex composition of alkaloids, sesquiterpene alkaloid dendrobine has been regarded as the quality standard for many Dendrobium plants (Li R. et al., 2017). Evidences from modern pharmacology have demonstrated that Dendrobium alkaloids have remarkable antihypertensive, anticancer, antipyretic, eye-benefiting, neuroprotective, and immune regulatory effects in preclinical studies (Wang J.H. et al., 2010; Li et al., 2011). Currently, alkaloids are primarily obtained through extraction and chemical synthesis (Kreis and Carreira, 2012). However, neither of these approaches is efficient enough because of extremely low accumulation levels in the Dendrobium plants and technical problems in total synthesis. Due to the high market demand, overexploitation, and deterioration of natural habitats, wild Dendrobium resources have been increasingly depleted. Therefore, the biotechnology-based strategy is promising for stably producing large quantities of alkaloids to meet the market demand and protect the wild resources.

\section{Dendrobium Alkaloid Biosynthesis}

One of the main purposes of transcriptome research in medicinal Dendrobium plants is to analyze the biosynthetic pathway and identify the key enzyme genes involved in specialized metabolite production. Several transcriptome studies have focused on the biosynthetic pathway of alkaloids in Dendrobium species. For instance, a previous study (Yuan et al., 2018) revealed that the alkaloids in the Dendrobium genus are mostly sesquiterpenoid alkaloids or terpenoid indole alkaloids (TIA). Functional analysis based on KEGG terms revealed 25 genes associated with alkaloid backbone construction that belonged to the TIA class by using the Roche 454 GS FLX Titanium platform (Guo et al., 2013; Figure 1). The upstream of the TIA pathway, which can be further divided into terpenoid-forming and indole pathway, is conserved among alkaloid-producing plants and initiated from the shikimate, mevolonate (MVA), or the methylerythritol phosphate (MEP) pathway (Figure 2). In the terpenoid-forming pathway, 10-hydroxylase (G10H) catalyzes geraniol to produce 10-hydroxygenraniol. After a series of enzymatic reactions, 10-hydroxygenraniol is converted to loganin, which is then further catalyzed by secologanin synthase (SCS) to generate secologanin (Wang C.T. et al., 2010). From the indole pathway, tryptamine is synthesized. These two intermediates, secologanin and tryptamine, then combined with each other by strictosidine synthase (STR) to form strictosidine (Zhu et al., 2014), a common precursor for all TIA biosynthesis.

The upstream of alkaloid biosynthesis is mainly through three pathways: the shikimate pathway, the MEP pathway, and the MVA pathway (Figure 2). The shikimate pathway in plants is 


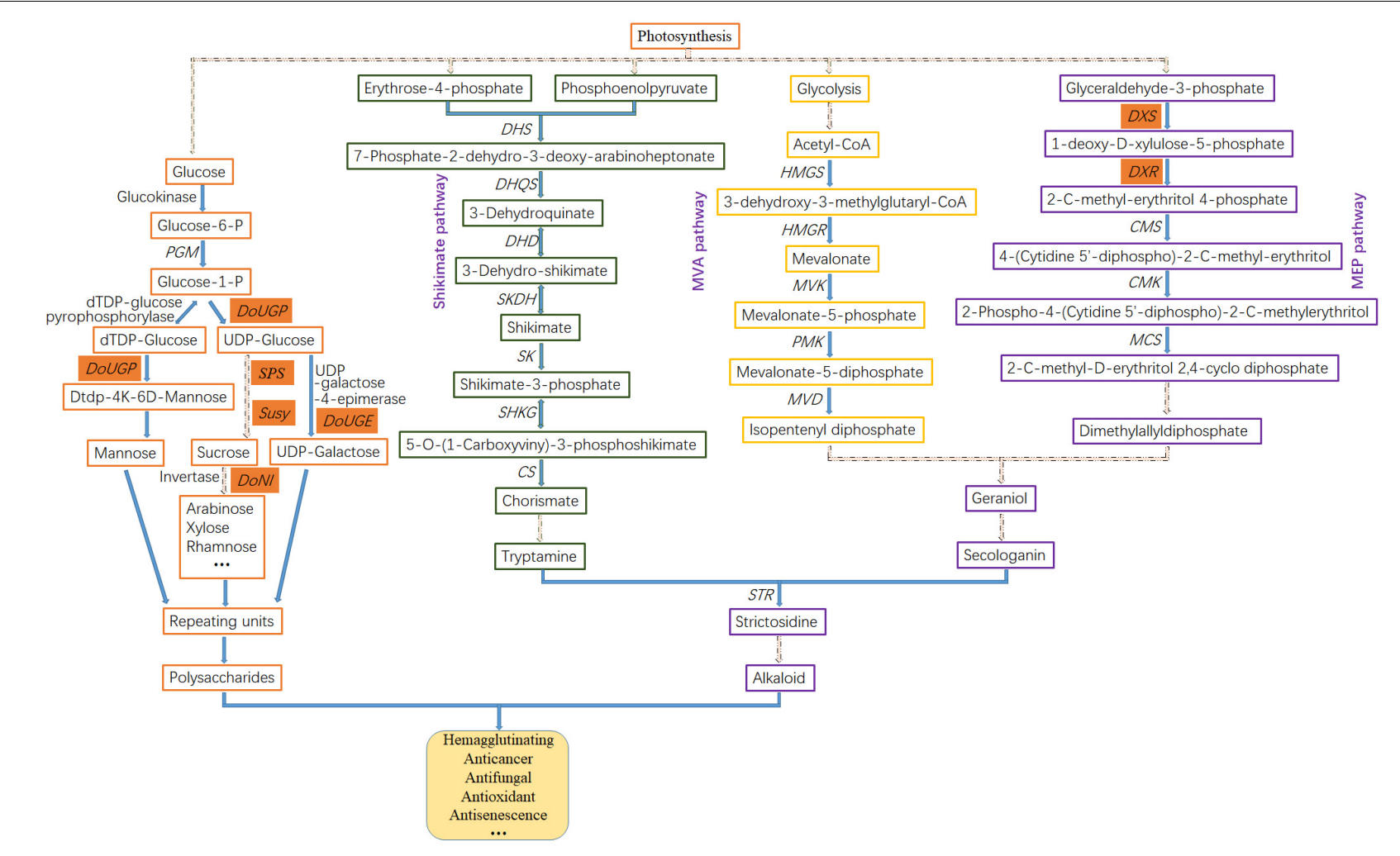

FIGURE 2 | Putative biosynthetic pathways of polysaccharide and alkaloid in Dendrobium. In the polysaccharide biosynthesis pathway, monosaccharides (mannose, glucose, galactose, arabinose, xylose, etc.) are produced through hydrolysis or hydrolysis-derivative reactions. These monosaccharides as basic building blocks and repeating units are then used to synthesize polysaccharides. In the alkaloid biosynthesis pathway, the upstream precursors are produced mainly through the Shikimate pathway, and MVA and MEP pathways. Genes in red cells indicate the cloned and functionally studied genes in Dendrobium. The dashed lines indicate multiple steps. Enzyme abbreviations are as follows: PGM, phosphoglucomutase; UGP, UDP-glucose pyrophosphorylase; SPS, sucrose-phosphate synthase; Susy, sucrose synthase; $D H S$, 3-dexoy-7-phosphoheptulonate synthase; $D H Q S$, 3-dehydroquinate synthase; $D H D$, 3-dehydroquinate dehydratase; $S K D H$, shikimate dehydrogenase; SK, shikimate kinase; SHKG, 3-phosphoshikimate 1-carboxyvinyltransferase; CS, chorismate synthase; HMGS, hydroxymethylglutaryl-CoA synthase; HMGR, 3-hydroxy-3-methylglutaryl CoA reductase; MVK, mevalonate kinase; PMK, phosphomevalonate kinase; MVD, diphosphomevalonate decarboxylase; DXS, 1-deoxy-D-xylulose-5-phosphate synthase; DXR, 1-deoxy-D-xylulose-5-phosphate reductoisomerase; CMS, 2-C-methyl-D-erythritol 4-phosphate cytidylyl transferase; CMK, 4-(cytidine 5'-diphospho)-2-C-methyl-D-erythritol kinase; MCS, 2-C-methyl-D-erythritol 2,4-cyclodiphosphate synthase; STR, strictosidine synthase.

essential for a variety of second metabolite synthesis (Tzin et al., 2012). A series of key enzymes involved in shikimate pathway have been identified. Among them, 5-enolpyruvylshikimate3-phosphate synthase (EPSP) is a key enzyme involved in the formation of enolpyruvylshikimate 3-phosphate (Klee et al., 1987). The stem-specific expression of EPSP in D. huoshanense enhanced the accumulation of tryptamine, which is a precursor for strictosidine biosynthesis (Yuan et al., 2018). Seventeen unigenes associated with six enzymes were revealed by transcriptome analysis in D. officinale and were mapped to the shikimate pathway (Shen et al., 2017), including 3deoxy-D-arabinoheptulosonate-7-phosphate synthase (DHS), 3-dehydroquinate synthase (DHQS), 3-dehydroquinate acid dehydratase (DHD), shikimate dehydrogenase (SKDH), 5enolpyruvylshikimate-3-phosphate synthase (SHKG), and farnesyl diphosphate synthase (FPS). In the MEP pathway, 1-deoxy-D-xylulose 5-phosphate synthase (DXS) is the first key enzyme, and 1-deoxy-D-xylulose-5-phosphate reductoisomerase (DXR) is the second and rate-limiting enzyme catalyzing a branched isovaleric precursor to form a straight chain pentose sugar (Ramak et al., 2013). Overexpression of DXS in Spike lavender or DXR in Salvia miltiorrhiza resulted in a significant increase in terpenoid accumulation (Munoz-Bertomeu et al., 2006). In the MVA pathway, 3-hydroxy-3-methyl-glutarylcoenzyme A reductase (HMGR) is one of the key enzymes involved in terpenoid biosynthesis. It is capable of catalyzing 3-hydroxy-3-methylglutaryl coenzyme A (HMG-CoA) to form MVA. Overexpression of HMGR1 in ginseng enhanced the steroid and triterpene production (Kim et al., 2014), suggesting the promoting role of HMGR1 in ginsenoside biosynthesis.

Previous reports revealed 34 alkaloids isolated from 14 Dendrobium species, 21 of which were dendrobine alkaloids with a sesquiterpene skeleton structure (Xu et al., 2017). A pharmacology study demonstrated that dendrobine restrains the growth of $A 549$ lung cancer cells and acts as a promising agent for treating virus infection ( $\mathrm{Li} \mathrm{R}$. et al., 2017). The upstream biosynthetic pathway of dendrobine is composed of the MVA and MEP pathway, which are conserved to 


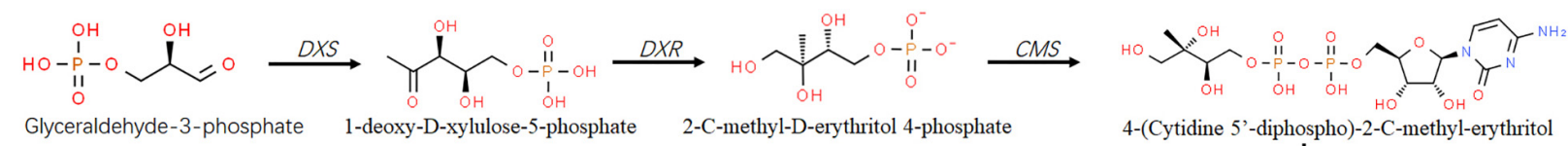

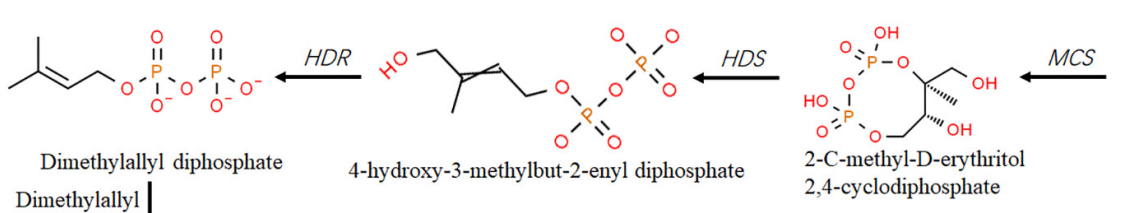

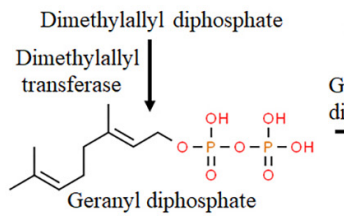

4-hydroxy-3-methylbut-2-enyl diphosphate

Geranyl diphosphate

diphosphatase

2-C-methyl-D-erythritol

4-(Cytidine 5'-diphospho)-2-C-methyl-erythritol

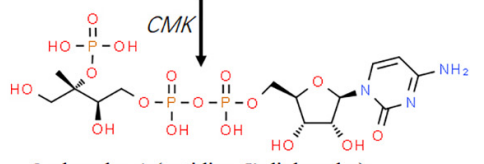

2-phospho-4-(cytidine 5'-diphospho)

-2-C-methyl-D-erythritol

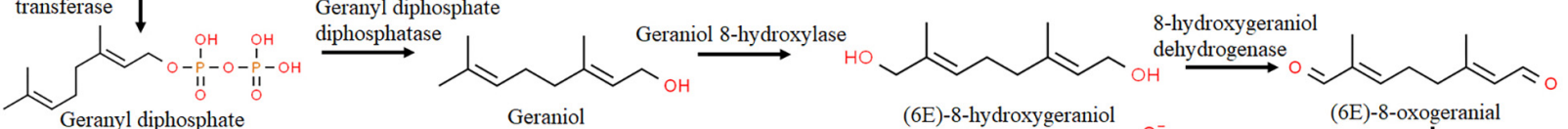

droxygeraniol

(6E)-8-oxogeranial
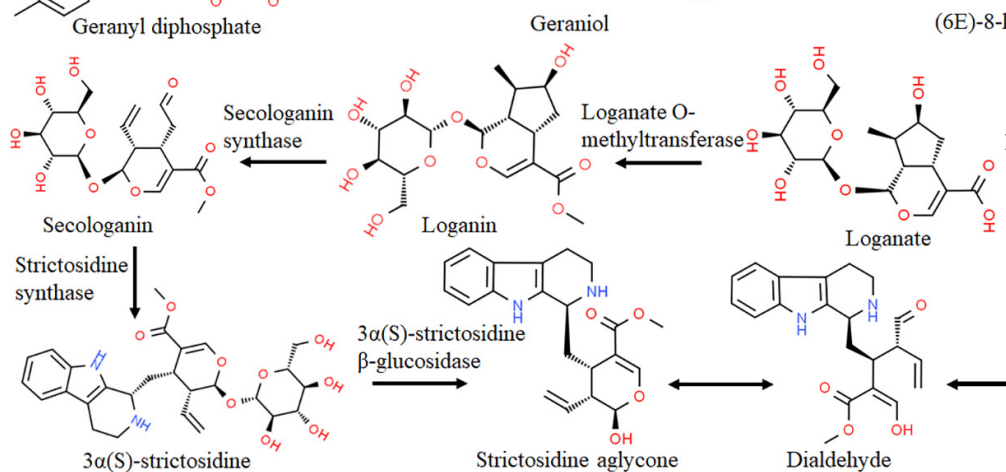$$
\text { . }
$$

Loganate

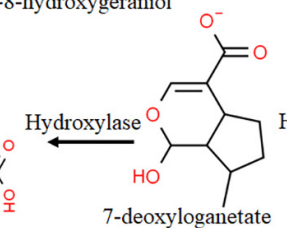

Hydroxyse
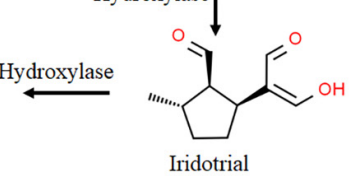

Iridotrial

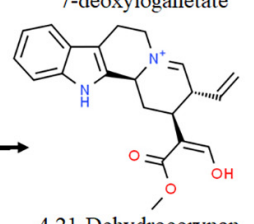

4,21-Dehydrocorynan -theine aldehyde

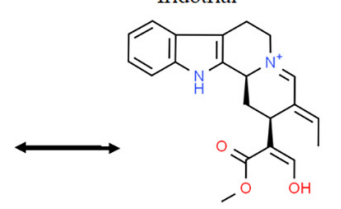

4,21-Dehydrogeissoschizine Geissoschizine dehydrogenase

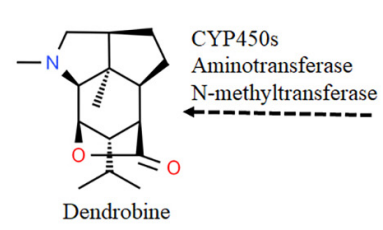

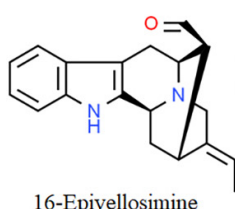

16-Epivellosimine
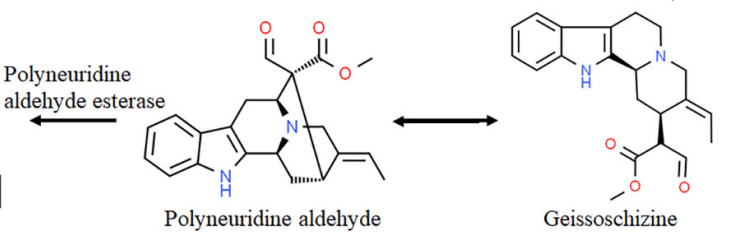

FIGURE 3 | Biosynthesis of dendrobine in Dendrobium through the MEP pathway. The putative pathway was adapted from Li Q. et al. (2017) and Zheng et al. (2018). The enzymes or enzyme encoding genes were indicated alongside the arrows. The dashed lines indicate multiple steps.

provide basic skeleton for terpenoid alkaloids (Figure 2). Both pathways can produce isopentenyl diphosphate (IPP), which is the precursor of synthetic terpenes that can be exchanged on the plasma membrane. Key enzymes involved in the MEP pathway (Figure 3) including DXS and DXR, as well as enzymes functioning in the MVA pathway such as hydroxymethylglutarylCoA synthase (HMGS) and HMGR have been annotated in D. officinale (Fan et al., 2016; Chen et al., 2019). In D. nobile, large-scale transcriptome analysis has been generated in response to MF23 infection with increased dendrobine production (Li R. et al., 2017). From the datasets, 16 genes encoding acetyl CoA acetyltransferase (AACT), phosphomevalonate kinase (PMK), diphosphomevalonate decarboxylase (MVD), and terpene synthase 21 (TPS21), which are members related to the biosynthesis of the backbone of sesquiterpene alkaloid dendrobine, have been identified, suggesting the dominant role of the MVA pathway in this process. In addition, 11 genes encoding nine enzymes were mapped onto the MEP pathway (Figure 3), which serves as a supplemental provider of isoprene units for dendrobine biosynthesis.

\section{Post-modifications}

Following the generation of strictosidine, the alkaloid biosynthesis in Dendrobium is characterized mainly by a set of post modifications (PTM), such as cytochrome P450s (CYP450s)-mediated oxidation and hydroxylation reactions (Guo et al., 2013). As a complex superfamily of monooxygenase, CYP450s play key roles in specialized metabolite biosynthesis, and some of them have been isolated and characterized (Seki et al., 2008). For instance, annotation of the 454 EST pool of $D$. officinale against the SwissProt database revealed 93 CYP450s transcripts belonging to 17 families (Guo et al., 2013). Among them, transcripts of the CYP71 (9.7\%) were likely to be involved in hydroxylation steps of alkaloid biosynthesis. In $D$. huoshanense, 229 unigenes were identified as putative CYP450s, most of which were also the CYP71 family members (7.8\%), followed by CYP3A and CYP4 family members (Yuan et al., 2018). Moreover, several post-modification enzymes involved in the biogenic pathway of dendrobine, such as CYP450s, aminotransferase, and methyltransferase (Figure 3), have been identified in D. nobile (Li Q. et al., 2017) and 
D. officinale (Chen et al., 2019). Among them, CYP1D10, METTL23, ATX4, and BCAT2 were significantly upregulated in response to MF23 infection (Li Q. et al., 2017), suggesting their positive roles in promoting dendrobine biosynthesis.

Transcription factor (TF) also plays a vital role in coordinating the expression of alkaloid biosynthesis genes, thus affecting the constituents' composition in higher plants (Table 3). For example, the AP2/ERF family members ORCA2 and ORCA3 were capable of regulating a subset of alkaloid biosynthesis genes (Van Der Fits and Memelink, 2000). Additionally, a large number of TFs, including C3H, bHLH, bZIP, MYB, and WRKY (Yuan et al., 2018), have been previously reported to play central roles in the regulation of alkaloid biosynthesis in Dendrobium.

\section{DENDROBIUM POLYSACCHARIDE BIOSYNTHESIS}

\section{Dendrobium Polysaccharide}

Many Dendrobium plants are precious medicinal herbs partially because of their abundant polysaccharides found in stems, leaves, and flowers. In recent years, many soluble polysaccharides were extracted from various Dendrobium species, and the structure, composition, and bioactivity were determined (He et al., 2015). The major polysaccharides in Dendrobium stems were non-starch mannan polysaccharides, to a lesser extent glucose, and a small amount of galactose (Ng et al., 2012). Semithin and ultrathin sections demonstrated that polysaccharides that formed granules were stored in the plastids of stems (He et al., 2017a), similar to starch grains.

\section{Dendrobium Polysaccharide Biosynthesis}

Polysaccharides are one of the major medicinal components in Dendrobium plants with antitumor, antioxidant, antiaging, antibacterial, antiviral, antiradiation, and anticoagulant activities (Liu et al., 2011; Wang et al., 2014). Thus far, a large number of carbohydrate-related genes (Li Q. et al., 2017) encoding glycosyltransferase (GT), glucosyltransferase, and mannosyltransferase, most of which were expressed more highly in stems than in leaves and roots, have been identified and might play vital roles in polysaccharide synthesis (Figure 2).

TABLE 3 | Transcription factor families identified in the medicinal Dendrobium transcriptome datasets.

\begin{tabular}{lcccc}
\hline $\begin{array}{l}\text { No. of unique } \\
\text { transcripts }\end{array}$ & $\begin{array}{r}\text { Guo et al., } \\
\mathbf{2 0 1 3}\end{array}$ & $\begin{array}{c}\text { Zhang J. } \\
\text { et al., 2016 }\end{array}$ & $\begin{array}{c}\text { Yuan et al., } \\
\mathbf{2 0 1 8}\end{array}$ & $\begin{array}{c}\text { Chen et al., } \\
\mathbf{2 0 1 9}\end{array}$ \\
\hline bHLH & 72 & 75 & 145 & 93 \\
bZIP & 49 & 23 & 227 & 23 \\
WRKY & 44 & 60 & 71 & 71 \\
MYB & 36 & 82 & 64 & 95 \\
NAC & 36 & 24 & 69 & 61 \\
GRAS & 24 & 4 & 37 & 15 \\
MADS & 13 & 30 & 28 & 25 \\
TCP & 13 & 6 & 24 & 25
\end{tabular}

Enzymes directly involved in polysaccharide metabolism, such as GDP-mannose pyrophosphorylase (GMP) in Dendrobium, have also been characterized (He et al., 2017c). Moreover, it has been confirmed that sucrose synthase (Susy) was positively correlated with the polysaccharide content in $D$. officinale and $D$. huoshanense and that two unique gene families, the galacturonosyltransferase and $\beta$-galactosidase gene families, were related to the richness of polysaccharides in D. officinale (Yan et al., 2015). GT is a group of carbohydrate-active enzymes that catalyzes the glycosidic bond formation in glycan and glycoside biosynthesis by transfer of sugar moieties from active donors to acceptor molecules (Shao et al., 2005; Lao et al., 2014). A recent transcriptome analysis in D. officinale (Shen et al., 2017) identified 280 GTs, including genes encoding glucosyltransferase (236), fucosyltransferase (11), mannosyltransferase (16), and xylosyltransferase (17) using BLASTX methods.

Mannan polysaccharides are the major component of polysaccharides from most of the Dendrobium species, accounting for as much as $58.3 \%$ of the dry weight of the crude polysaccharide fraction in D. officinale (Xing et al., 2015), and also promising bioactive ingredients for use in drugs. It has beneficial effects on human health with increased cytokine production and antioxidant and anticancer activities (Xing et al., 2015). Mannan polysaccharides can be further classified into four subfamilies (Buckeridge, 2010): pure mannan, glucomannan (GM), galactomannan (GGM), and galactoglucomannan. The biosynthesis of mannan polysaccharides is mediated by mannan synthases using GDP-D-mannose or GDP-D-glucose as substrates (Hassid, 1969). Moreover, the cellulose synthase A (CesA) superfamily genes have also been demonstrated to be involved in the biosynthesis of mannan polysaccharides (Lerouxel et al., 2006). The CesA superfamily can be subdivided into one cellulose synthase (CesA) family and nine cellulose synthase-like (Csl) families, CslA to CslJ (Suzuki et al., 2006). In D. officinale, eight CslA genes (DoCslA1 to DoCslA8) were identified and analyzed to provide genetic evidences for their roles in mannan polysaccharide biosynthesis (He et al., 2017a). Overexpression of DoCslA6 increased mannose content in Arabidopsis thaliana (He et al., 2017a). In addition, GDPmannose transporter (GMT), which translocates GDP-mannose into the Golgi lumen, is indispensable for mannan polysaccharide biosynthesis. Three GMT genes, DoGMT1 to DoGMT3 (Yu et al., 2018), have been identified in D. officinale with the highest transcript levels in stems.

\section{Polysaccharide Synthesis and Sucrose Metabolism}

Polysaccharide synthesis and sucrose metabolism are closely linked because many monosaccharides, the basic building blocks for polysaccharide synthesis, are produced from sucrose hydrolysis or hydrolysis derivatives. In general, sucrose metabolism involves two distinct processes, sucrose synthesis and sucrose breakdown, which are mainly catalyzed by sucrose phosphate synthase (SPS) and Susy, respectively (Huber and Huber, 1996). A previous study revealed that the levels of polysaccharides in $D$. officinale were closely related to the 
concentrations of the reduced sugar and soluble sugar, which were directly affected by sucrose invertase and SPS activities (Yan et al., 2015). Yan Liang and colleagues (Yan et al., 2015) analyzed the genome sequence of $D$. officinale and identified 10 SPS and 15 Susy genes, which have undergone marked expansion through tandem duplication. Likewise, in D. huoshanense, 13 SPS and 18 Susy genes have been isolated (Yuan et al., 2018). Alkaline/neutral invertase (NI) is responsible for sucrose hydrolysis to produce glucose or fructose in the cytoplasm. An NI gene, DoNI, was cloned in D. officinale by the rapid amplification of cDNA ends (RASE) method. The expression of DoNI was associated with its activities in different tissues and, more importantly, the polysaccharide accumulation (Gao et al., 2016). Uridine diphosphate glucose (UDPG) is an important direct or indirect glycosyl donor for synthesis of polysaccharides. The UDPG pyrophosphorylase (UDPase) is a key enzyme for reversibly catalyzing UDPG into glucose-1-phosphate (Glc-P), which is then utilized in synthesis of polysaccharides by GTs. A novel UGPase gene, DoUGP, was identified from D. officinale (Wan et al., 2017). It was highly expressed in stems in comparison to other organs and positively correlated with the highest polysaccharide content there (Wan et al., 2017). Sucrose feeding significantly increased DoUGP expression and enhanced polysaccharide production accordingly in both protocorm of D. officinale and protocorm-like bodies of $D$. huoshanense in suspension cultures (Wan et al., 2017). Thus, DoUGP is probably involved in polysaccharide synthesis and might serve as a potential target for quality breeding of Dendrobium orchids.

\section{BIOSYNTHESIS OF OTHER COMPOUNDS IN DENDROBIUM}

\section{Tropine Biosynthesis-Related Genes}

Tropine is an alkaloid derived from tropinone, which can be reduced by tropinone reductase (TRs) using the NADPH as coenzyme (Nakajima et al., 1998). TRs can be further divided into two subgroups, TRI and TRII, based on the stereospecificity of reduction product. TRI is responsible for tropine production, whereas TRII is mainly involved in the generation of pseudotropine (Nakajima et al., 1998). Most of $T R$ homologous genes found in other plant species do not have tropinone reduction activity, except for plants belonging to or closely related to the Solanaceae family (Drager, 2006) and CoTR from Cochlearia officinalis (Brock et al., 2008). Recently, DnTR1 and DnTR2 that encode peptides with similarity to known TRs were cloned from $D$. nobile. Catalytic activity assay revealed that both DnTR1 and DnTR2 were able to reduce 3-quinuclidinone hydrochloride and 4-methylcyclohexanone using NADPH as coenzyme (Chen et al., 2013). Moreover, DnTR1 could reduce tropinone, whereas DnTR2 couldn't (Cheng et al., 2013), implying their tremendous variation in substrate specificity.

\section{Flavonoid Biosynthesis-Related Genes}

Flavonoids are the second most common compounds in D. officinale, exhibiting diverse medicinal functions including antioxidant and protective effects on cell toxicity and treatment of various degenerative and age-related diseases (Prochazkova et al., 2011; Wang et al., 2017). Most flavonoids in Dendrobium are C-glycosides with basic skeletons including vitexin, quercetin, luteolin, apigenin, etc. (Lei et al., 2018). The biosynthesis of most flavonoids begins in the phenylpropanoid pathway using malonyl-CoA and p-coumaroyl-CoA as precursors (Liu et al., 2013). The whole processes are regulated by many key enzymes, transcription factors, UDP-GT, and CYP450s (Liu et al., 2013). Transcriptome analysis (Lei et al., 2018) revealed that 31 unigenes encoding 14 enzymes were involved in the biosynthesis of flavonoids in D. catenatum. Synthesis of the three basic flavonoid glycoside skeletons are regulated by flavonol synthase (FLS), CYP75A, and flavonoid 3'-monooxygenase. Specifically, FLS is involved in the transformation of dihydroquercetin to quercetin, and dihydrokaempferol to kaempferol, which can be subsequently catalyzed into quercetin by CYP75A and flavonoid 3 '-monooxygenase (Lei et al., 2018), two enzymes also regulating the synthesis of luteolin from apigenin.

\section{STRATEGIES FOR ENHANCING DENDROBIUM BIOACTIVE COMPOUNDS PRODUCTION}

Elicitation and precursor feeding are two major effective methods for increasing the accumulation of specialized metabolites (Skrzypczak-Pietraszek et al., 2014). Mycorrhizal fungi isolated from the roots of wild $D$. officinale and $D$. nobile can serve as an elicitor for seed germination and specialized metabolite production (Xu et al., 2014), by offering nutrients such as glucose directly to their hosts, or through secreting certain types of phytohormones supplied to the hosts (Zhang, 1999). For instance, inoculation of $D$. nobile with MF23, a mycorrhizal fungus previously isolated from the roots of D. officinale, significantly increased total alkaloid content $(18.3 \%)$ by forming peloton to supply nutrients for their hosts (Zhang et al., 2012). Similarly, Ceratocystis fimbriata infection significantly increased alkaloid accumulation in mango (Araujo et al., 2016).

Apart from the biotic factors, some abiotic stresses induced by unfavorable environments (drought, salt, etc.) and stress hormones (methyl jasmonate, MeJA) can also promote specialized metabolite accumulation. For example, the alkaloid biosynthesis was markedly increased in Catharanthus roseus and Motherwort by binary stress and drought stress, respectively (Wei et al., 2013; Zhu et al., 2015). The phytohormone MeJA has been identified as a signaling molecule that switches on gene expression and enhances the biosynthesis of various bioactive compounds, particularly alkaloids and polysaccharides in medicinal plants including Dendrobium species (Zhan et al., 2018; Zhang et al., 2018). Exogenous feeding of MeJA enhanced the catalytic efficiency and the expression of strictosidine synthase (STR), which plays a vital role in alkaloid biosynthesis (Paul et al., 2017). Additionally, precursor feeding has also been performed to increase the alkaloid/polysaccharide production, 
such as tryptamine application to enhance reserpine synthesis in Rauvolfia serpentina; tryptophan, tryptamine, secologanin, and loganin feeding to promote the accumulation of ajmalicine, vindoline, and catharanthine in C. roseus (Panwar and Guru, 2015). Likewise, sucrose feeding (Wan et al., 2017) upregulated DoUGP transcription, and correspondingly increased polysaccharides content in D. officinale.

\section{PERSPECTIVES}

Dendrobium genus in Orchidaceae is well known worldwide for its high economic and medicinal values. Recently, transcriptomes of Dendrobium have been sequenced for validation of genes involved in specialized metabolite biosynthesis. The resultant datasets will contribute to further research on metabolic pathways, molecular genetic breeding, genetic engineering, excavation, and protection of genetic resources of medicinal Dendrobium plants. Along with the progress of sequencing technology, novel strategies of targeted isolation, purification, nuclear magnetic resonance (NMR) identification, proteomics, and metabolomics will facilitate the full exploration of the molecular mechanism of bioactive ingredient biosynthesis regulation (Figure 4). Meanwhile, a great many of the genes associated with post-modifications (PTMs) have been identified. They are complex but evolutionarily conserved biochemical modifications consisting of hundreds of directly or indirectly intertwined reactions in various eukaryotic and prokaryotic cells. Although transcriptome analysis revealed that the expression levels of some genes encoding post-modification enzymes, such as CYP450s, aminotransferases, and methyltransferases were upregulated during bioactive compounds production, future research regarding the downstream of PTM processes should be performed. Moreover, abiotic (cold acclimation, light intensity,

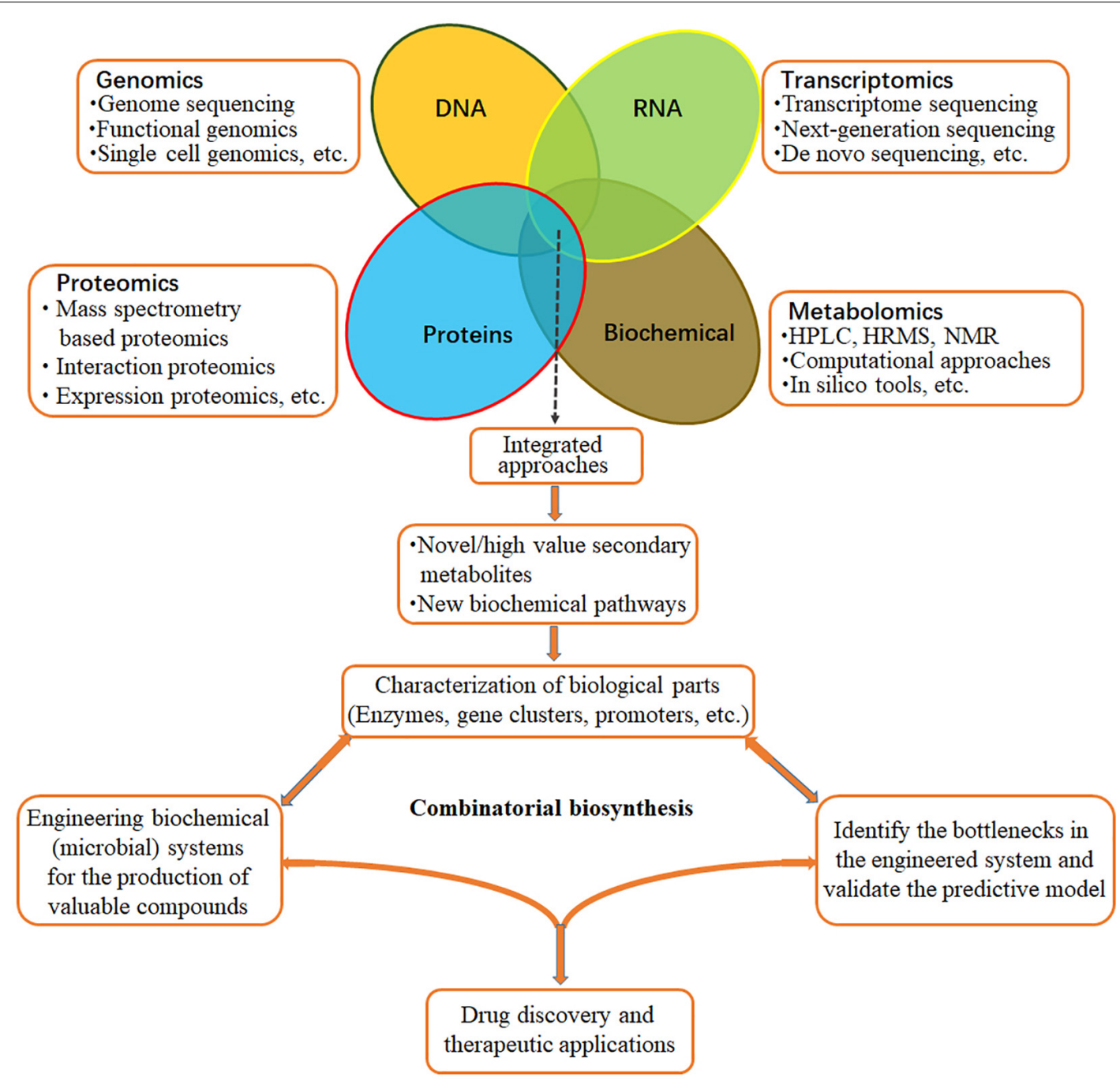

FIGURE 4 | Integrated approaches for production of bioactive medicinal ingredients. Combining multiple "omics" datasets will facilitate the discovery of novel bioactive specialized metabolites and characterization of the related biosynthesis pathways in Dendrobium species. Based on the obtained knowledge, novel combinatorial biosynthesis systems can be engineered for the production of new and interesting specialized metabolites. 
water, salt stress, etc.) and biotic (fungus infection) stresses and precursor feeding can significantly influence the expression of numerous genes involved in metabolome and the accumulation of metabolites in Dendrobium. Therefore, it is feasible to improve active ingredients production by the combinations of stress stimulation and precursor feeding. Even though the engineering of specialized metabolite production is quite challenging due to incomplete and uncertain information, combinatorial biosynthesis (Figure 4) that reconstitutes genes from plant metabolomic pathways in microorganisms or other plant species for the targeted ingredients biosynthesis, or combines genes from different microorganisms for the production of new and interesting plant specialized metabolites is of great potential for further investigation.

\section{REFERENCES}

An, H., Zhu, Q., Pei, W., Fan, J., Liang, Y., Cui, Y., et al. (2016). Wholetranscriptome selection and evaluation of internal reference genes for expression analysis in protocorm development of Dendrobium officinale Kimura et Migo. PLoS One 11:e0163478. doi: 10.1371/journal.pone.0163478/ 1932-6203

Araujo, L., Bispo, W. M. S., Rios, J., Fernandes, S., and Rodrigues, F. (2016). Alkaloids and phenolics biosynthesis increases mango resistance to infection by Ceratocystis fimbriata. Bragantia 75, 199-211. doi: 10.1590/1678-4499.261

Brock, A., Brandt, W., and Drager, B. (2008). The functional divergence of shortchain dehydrogenases involved in tropinone reduction. Plant J. 54, 388-401. doi: 10.1111/j.1365-313X.2008.03422.x/1365-313X

Buckeridge, M. S. (2010). Seed cell wall storage polysaccharides: models to understand cell wall biosynthesis and degradation. Plant Physiol. 154, 10171023. doi: 10.1104/pp.110.158642/0032-0889

Chen, W., Cheng, X., Zhou, Z., Liu, J., and Wang, H. (2013). Molecular cloning and characterization of a tropinone reductase from Dendrobium nobile Lindl. Mol. Biol. Rep. 40, 1145-1154. doi: 10.1007/s11033-012-2156-0/15734978

Chen, Y., Wang, Y., Lyu, P., Chen, L., Shen, C., and Sun, C. (2019). Comparative transcriptomic analysis reveal the regulation mechanism underlying MeJAinduced accumulation of alkaloids in Dendrobium officinale. J. Plant Res. 132, 419-429. doi: 10.1007/s10265-019-01099-6/1618-0860

Cheng, X., Chen, W., Zhou, Z., Liu, J., and Wang, H. (2013). Functional characterization of a novel tropinone reductase-like gene in Dendrobium nobile Lindl. J. Plant Physiol. 170, 958-964. doi: 10.1016/j.jplph.2013.02.007/16181328

Drager, B. (2006). Tropinone reductases, enzymes at the branch point of tropane alkaloid metabolism. Phytochemistry 67, 327-337. doi: 10.1016/j.phytochem. 2005.12.001/0031-9422

Fan, H., Wu, Q., Wang, X., Wu, L., Cai, Y., and Lin, Y. (2016). Molecular cloning and expression of 1-deoxy-D-xylulose-5-phosphate synthase and 1-deoxy-Dxylulose-5-phosphate reductoisomerase in Dendrobium officinale. Plant Cell Tissue Organ Cult 125, 381-385. doi: 10.1007/s11240-016-0945-1

Gao, F., Cao, X. F., Si, J. P., Chen, Z. Y., and Duan, C. L. (2016). Characterization of the alkaline/neutral invertase gene in Dendrobium officinale and its relationship with polysaccharide accumulation. Genet. Mol. Res. 15:gmr7647. doi: 10.4238/ gmr.15027647/1676-5680

Guo, X., Li, Y., Li, C., Luo, H., Wang, L., Qian, J., et al. (2013). Analysis of the Dendrobium officinale transcriptome reveals putative alkaloid biosynthetic genes and genetic markers. Gene 527, 131-138. doi: 10.1016/j.gene.2013.05.073/ 1879-0038

Hassid, W. Z. (1969). Biosynthesis of oligosaccharides and polysaccharides in plants. Science 165, 137-144. doi: 10.1126/science.165.3889.137/0036-8075

He, C., Wu, K., Zhang, J., Liu, X., Zeng, S., Yu, Z., et al. (2017a). Cytochemical localization of polysaccharides in Dendrobium officinale and the involvement of DoCSLA6 in the synthesis of mannan polysaccharides. Front. Plant Sci. 8:173. doi: 10.3389/fpls.2017.00173/1664-462X

\section{AUTHOR CONTRIBUTIONS}

ZW and MW conceived the project. ZW drafted the manuscript. $\mathrm{ZW}, \mathrm{MZ}$, and $\mathrm{HC}$ evaluated and interpreted the data. JL and MW revised the manuscript. All authors read and approved the final manuscript.

\section{FUNDING}

This work was financially supported by the Forestry Science and Technology Innovation Project of Guangdong Province (2017KJCX062) and the Medical Science and Technology Research Fund Project of Guangdong Province (A2019093).

He, L., Fu, S., Xu, Z., Yan, J., Xu, J., Zhou, H., et al. (2017b). Hybrid sequencing of full-length cDNA transcripts of stems and leaves in Dendrobium officinale. Genes 8:257. doi: 10.3390/genes8100257

He, C., Yu, Z., Teixeira da Silva, J. A., Zhang, J., Liu, X., Wang, X., et al. (2017c). DoGMP1 from Dendrobium officinale contributes to mannose content of watersoluble polysaccharides and plays a role in salt stress response. Sci. Rep. 7:41010. doi: 10.1038/srep41010

He, C., Zhang, J., Liu, X., Zeng, S., Wu, K., Yu, Z., et al. (2015). Identification of genes involved in biosynthesis of mannan polysaccharides in Dendrobium officinale by RNA-seq analysis. Plant Mol. Biol. 88, 219-231. doi: 10.1007/ s11103-015-0316-z/1573-5028

Hu, J. M., Chen, J. J., Yu, H., Zhao, Y. X., and Zhou, J. (2008). Five new compounds from Dendrobium longicornu. Planta Med. 74, 535-539. doi: 10.1055/s-20081074492/0032-0943

Huber, S. C., and Huber, J. L. (1996). Role and regulation of sucrose-phosphate synthase in higher plants. Annu. Rev. Plant Physiol. Plant Mol. Biol. 47, 431-444. doi: 10.1146/annurev.arplant.47.1.431/1040-2519

Ito, M., Matsuzaki, K., Wang, J., Daikonya, A., Wang, N. L., Yao, X. S., et al. (2010). New phenanthrenes and stilbenes from Dendrobium loddigesii. Chem. Pharm. Bull. 58, 628-633. doi: 10.1248/cpb.58.628/0009-2363

Jin, J., Liang, Y., Xie, H., Zhang, X., Yao, X., and Wang, Z. (2008). Dendroflorin retards the senescence of MRC-5 cells. Pharmazie 63, 321-323.

Kim, Y. J., Lee, O. R., Oh, J. Y., Jang, M. G., and Yang, D. C. (2014). Functional analysis of 3-hydroxy-3-methylglutaryl coenzyme a reductase encoding genes in triterpene saponin-producing ginseng. Plant Physiol. 165, 373-387. doi: 10. 1104/pp.113.222596/1532-2548

Klee, H. J., Muskopf, Y. M., and Gasser, C. S. (1987). Cloning of an Arabidopsis thaliana gene encoding 5-enolpyruvylshikimate-3-phosphate synthase: sequence analysis and manipulation to obtain glyphosate-tolerant plants. Mol. Gen. Genet. 210, 437-442. doi: 10.1007/bf00327194/0026-8925

Kreis, L. M., and Carreira, E. M. (2012). Total synthesis of (-)-dendrobine. Angew. Chem. Int. Ed. Engl. 51, 3436-3439. doi: 10.1002/anie.201108564/1521-3773

Lao, J., Oikawa, A., Bromley, J. R., Mcinerney, P., Suttangkakul, A., Smith-Moritz, A. M., et al. (2014). The plant glycosyltransferase clone collection for functional genomics. Plant J. 79, 517-529. doi: 10.1111/tpj.12577/0960-7412

Lei, Z., Zhou, C., Ji, X., Wei, G., Huang, Y., Yu, W., et al. (2018). Transcriptome analysis reveals genes involved in flavonoid biosynthesis and accumulation in Dendrobium catenatum from different locations. Sci. Rep. 8:6373. doi: 10.1038/ s41598-018-24751-y/2045-2322

Lerouxel, O., Cavalier, D. M., Liepman, A. H., and Keegstra, K. (2006). Biosynthesis of plant cell wall polysaccharides - a complex process. Curr. Opin. Plant Biol. 9, 621-630. doi: 10.1016/j.pbi.2006.09.009/1369-5266

Li, Q., Ding, G., Li, B., and Guo, S. X. (2017). Transcriptome analysis of genes involved in dendrobine biosynthesis in Dendrobium nobile Lindl. infected with mycorrhizal fungus MF23 (Mycena sp.). Sci. Rep. 7:316. doi: 10.1038/s41598017-00445-9/2045-2322

Li, R., Liu, T., Liu, M., Chen, F., Liu, S., and Yang, J. (2017). Anti-influenza A virus activity of dendrobine and its mechanism of action. J. Agric. Food Chem. 65, 3665-3674. doi: 10.1021/acs.jafc.7b00276/1520-5118 
Li, Y., Li, F., Gong, Q., Wu, Q., and Shi, J. (2011). Inhibitory effects of Dendrobium alkaloids on memory impairment induced by lipopolysaccharide in rats. Planta Med. 77, 117-121. doi: 10.1055/s-0030-1250235/0032-0943

Liu, X., Lu, Y., Yuan, Y., Liu, S., Guan, C., Chen, S., et al. (2013). De novo transcriptome of Brassica juncea seed coat and identification of genes for the biosynthesis of flavonoids. PLoS One 8:e71110. doi: 10.1371/journal.pone. 0071110/1932-6203

Liu, X. F., Zhu, J., Ge, S. Y., Xia, L. J., Yang, H. Y., Qian, Y. T., et al. (2011). Orally administered Dendrobium officinale and its polysaccharides enhance immune functions in BALB/c mice. Nat. Prod. Commun. 6, 867-870.

Lu, T. L., Han, C. K., Chang, Y. S., Lu, T. J., Huang, H. C., Bao, B. Y., et al. (2014). Denbinobine, a phenanthrene from Dendrobium nobile, impairs prostate cancer migration by inhibiting Rac1 activity. Am. J. Chin. Med. 42, 1539-1554. doi: 10.1142/s0192415x14500967/0192-415x

Luo, A., He, X., Zhou, S., Fan, Y., He, T., and Chun, Z. (2009). In vitro antioxidant activities of a water-soluble polysaccharide derived from Dendrobium nobile Lindl. extracts. Int. J. Biol. Macromol. 45, 359-363. doi: 10.1016/j.ijbiomac.2009. 07.008/1879-0003

Munoz-Bertomeu, J., Arrillaga, I., Ros, R., and Segura, J. (2006). Up-regulation of 1-deoxy-D-xylulose-5-phosphate synthase enhances production of essential oils in transgenic spike lavender. Plant Physiol. 142, 890-900. doi: 10.1104/pp.106. 086355/0032-0889

Nakajima, K., Yamashita, A., Akama, H., Nakatsu, T., Kato, H., Hashimoto, T., et al. (1998). Crystal structures of two tropinone reductases: different reaction stereospecificities in the same protein fold. Proc. Natl. Acad. Sci. U.S.A. 95, 4876-4881. doi: 10.1073/pnas.95.9.4876/0027-8424

Ng, T. B., Liu, J., Wong, J. H., Ye, X., Wing, S., Tong, Y., et al. (2012). Review of research on Dendrobium, a prized folk medicine. Appl. Microbiol. Biotechnol. 93, 1795-1803. doi: 10.1007/s00253-011-3829-7/1432-0614

Pan, L. H., Lu, J., Luo, J. P., Zha, X. Q., and Wang, J. H. (2012). Preventive effect of a galactoglucomannan (GGM) from Dendrobium huoshanense on seleniuminduced liver injury and fibrosis in rats. Exp. Toxicol. Pathol. 64, 899-904. doi: 10.1016/j.etp.2011.04.001/0940-2993

Panwar, G. S., and Guru, S. K. (2015). Stimulation of reserpine production in the whole plant culture of Rauwolfia serpentina L. by elicitors and precursor feeding. J. Plant Biochem. Biotechnol. 24, 49-55. doi: 10.1007/s13562-013-02355

Paul, P., Singh, S. K., Patra, B., Sui, X., Pattanaik, S., and Yuan, L. (2017). A differentially regulated AP2/ERF transcription factor gene cluster acts downstream of a MAP kinase cascade to modulate terpenoid indole alkaloid biosynthesis in Catharanthus roseus. New Phytol. 213, 1107-1123. doi: 10.1111/ nph.14252/1469-8137

Pridgeon, A. M., Cribb, P. J., Chase, M. W., and Rasmussen, F. N. (2014). Genera Orchidacearum Volume 6: Epidendroideae. England: Oxford University Press.

Prochazkova, D., Bousova, I., and Wilhelmova, N. (2011). Antioxidant and prooxidant properties of flavonoids. Fitoterapia 82, 513-523. doi: 10.1016/j. fitote.2011.01.018/1873-6971

Ramak, P., Kazempour Osaloo, S., Ebrahimzadeh, H., Sharifi, M., and Behmanesh, M. (2013). Inhibition of the mevalonate pathway enhances carvacrol biosynthesis and DXR gene expression in shoot cultures of Satureja khuzistanica Jamzad. J. Plant Physiol. 170, 1187-1193. doi: 10.1016/j.jplph.2013. 03.013/1618-1328

Sattayasai, N., Sudmoon, R., Nuchadomrong, S., Chaveerach, A., Kuehnle, A. R., Mudalige-Jayawickrama, R. G., et al. (2009). Dendrobium findleyanum agglutinin: production, localization, anti-fungal activity and gene characterization. Plant Cell Rep. 28, 1243-1252. doi: 10.1007/s00299-0090724-0/0721-7714

Seki, H., Ohyama, K., Sawai, S., Mizutani, M., Ohnishi, T., Sudo, H., et al. (2008). Licorice beta-amyrin 11-oxidase, a cytochrome P450 with a key role in the biosynthesis of the triterpene sweetener glycyrrhizin. Proc. Natl. Acad. Sci. U.S.A. 105, 14204-14209. doi: 10.1073/pnas.0803876105/1091-6490

Shao, H., He, X., Achnine, L., Blount, J. W., Dixon, R. A., and Wang, X. (2005). Crystal structures of a multifunctional triterpene/flavonoid glycosyltransferase from Medicago truncatula. Plant Cell 17, 3141-3154. doi: 10.1105/tpc.105. 035055/1040-4651

Shen, C., Guo, H., Chen, H., Shi, Y., Meng, Y., Lu, J., et al. (2017). Identification and analysis of genes associated with the synthesis of bioactive constituents in Dendrobium officinale using RNA-Seq. Sci. Rep. 7:187. doi: 10.1038/s41598017-00292-8/2045-2322
Skrzypczak-Pietraszek, E., Slota, J., and Pietraszek, J. (2014). The influence of L-phenylalanine, methyl jasmonate and sucrose concentration on the accumulation of phenolic acids in Exacum affine Balf. f. ex Regel shoot culture. Acta Biochim. Pollut. 61, 47-53.

Song, J. I., Kang, Y. J., Yong, H. Y., Kim, Y. C., and Moon, A. (2012). Denbinobine, a phenanthrene from Dendrobium nobile, inhibits invasion and induces apoptosis in SNU-484 human gastric cancer cells. Oncol. Rep. 27, 813-818. doi: 10.3892/ or.2011.1551/1791-2431

Suzuki, S., Li, L., Sun, Y. H., and Chiang, V. L. (2006). The cellulose synthase gene superfamily and biochemical functions of xylem-specific cellulose synthase-like genes in Populus trichocarpa. Plant Physiol. 142, 1233-1245. doi: 10.1104/pp. 106.086678/0032-0889

Tsai, A. C., Pan, S. L., Liao, C. H., Guh, J. H., Wang, S. W., Sun, H. L., et al. (2010). Moscatilin, a bibenzyl derivative from the India orchid Dendrobrium loddigesii, suppresses tumor angiogenesis and growth in vitro and in vivo. Cancer Lett. 292, 163-170. doi: 10.1016/j.canlet.2009.11.020/0304-3835

Tzin, V., Malitsky, S., Ben, Z. M., Bedair, M., Sumner, L., Aharoni, A., et al. (2012). Expression of a bacterial feedback-insensitive 3-deoxy-D-arabinoheptulosonate 7-phosphate synthase of the shikimate pathway in Arabidopsis elucidates potential metabolic bottlenecks between primary and secondary metabolism. New Phytol. 194, 430-439. doi: 10.1111/j.1469-8137.2012.04052. $\mathrm{x} / 1469-8137$

Van Der Fits, L., and Memelink, J. (2000). ORCA3, a jasmonate-responsive transcriptional regulator of plant primary and secondary metabolism. Science 289, 295-297. doi: 10.1126/science.289.5477.295/0036-8075

Wan, R., Sun, J., He, T., Hu, Y., Zhao, Y., Wu, Y., et al. (2017). Cloning cDNA and functional characterization of UDP-glucose pyrophosphorylase in Dendrobium officinale. Biol. Plantarum 61, 147-154. doi: 10.1007/s10535-016-0645-Z

Wang, C. T., Liu, H., Gao, X. S., and Zhang, H. X. (2010). Overexpression of G10H and ORCA 3 in the hairy roots of Catharanthus roseus improves catharanthine production. Plant Cell Rep. 29, 887-894. doi: 10.1007/s00299-010-0874-0/ 1432-203X

Wang, J. H., Zha, X. Q., Luo, J. P., and Yang, X. F. (2010). An acetylated galactomannoglucan from the stems of Dendrobium nobile Lindl. Carbohydr. Res. 345, 1023-1027. doi: 10.1016/j.carres.2010.03.005/1873-426X

Wang, Q., Gong, Q., Wu, Q., and Shi, J. (2010). Neuroprotective effects of Dendrobium alkaloids on rat cortical neurons injured by oxygen-glucose deprivation and reperfusion. Phytomedicine 17, 108-115. doi: 10.1016/j. phymed.2009.05.010/1618-095X

Wang, D., Du, N., Wen, L., Zhu, H., Liu, F., Wang, X., et al. (2017). An efficient method for the preparative isolation and purification of flavonoid glycosides and caffeoylquinic acid derivatives from leaves of Lonicera japonica Thunb. using high speed counter-current chromatography (HSCCC) and PrepHPLC guided by DPPH-HPLC experiments. Molecules 22:E229. doi: 10.3390/ molecules22020229/1420-3049

Wang, H. Z., Feng, S. G., Lu, J. J., Shi, N. N., and Liu, J. J. (2009). Phylogenetic study and molecular identification of 31 Dendrobium species using inter-simple sequence repeat (ISSR) markers. Sci. Hortic 122, 440-447. doi: 10.1016/j.scienta. 2009.06.005

Wang, Z., Wang, C., Su, T., and Zhang, J. (2014). Antioxidant and immunological activities of polysaccharides from Gentiana scabra Bunge roots. Carbohydr. Polym. 112, 114-118. doi: 10.1016/j.carbpol.2014.05.077/0144-8617

Wei, H., Li, L., Yan, X., and Wang, Y. (2013). Effects of soil drought stress on the accumulation of alkaloids and flavonoids in motherwort. Adv. Inform. Sci. Serv. Sci. 5, 795-803. doi: 10.4156/aiss.vol5.issue6.94

Wu, K. G., Li, T. H., Chen, C. J., Cheng, H. I., and Wang, T. Y. (2011). A pilot study evaluating the clinical and immunomodulatory effects of an orally administered extract of Dendrobium huoshanense in children with moderate to severe recalcitrant atopic dermatitis. Int. J. Immunopathol. Pharmacol. 24, 367-375. doi: 10.1177/039463201102400210/0394-6320

Xing, X., Cui, S. W., Nie, S., Phillips, G. O., Goff, H. D., and Wang, Q. (2015). Study on Dendrobium officinale O-acetyl-glucomannan (Dendronan(R)): part II. Fine structures of O-acetylated residues. Carbohydr. Polym. 117, 422-433. doi: 10.1016/j.carbpol.2014.08.121/1879-1344

Xu, W., Dong, W., Bai, Y., Li, Z., Sun, W., Yang, S., et al. (2014). Growth promoting of Dendrobium endophytic fungus on Dendrobium officinale tissue culture seedlings. Southwest China J. Agr. Sci. 27, 317-324.

Xu, X. L., Qing, L., and Li, B. (2017). Review of research on polysaccharides and dendrobine of Dendrobium nobile Lindl. Res. Rev. 6, 54-56. 
Yan, L., Wang, X., Liu, H., Tian, Y., Lian, J., Yang, R., et al. (2015). The genome of Dendrobium officinale illuminates the biology of the important traditional Chinese orchid herb. Mol. Plant 8, 922-934. doi: 10.1016/j.molp.2014.12.011/ 1674-2052

Yang, H., Chou, G. X., Wang, Z. T., Hu, Z. B., and Xu, L. S. (2004). Two new fluorenones from Dendrobium chrysotoxum. J. Asian. Nat. Prod. Res. 6, 35-38. doi: 10.1080/1028602031000119790/1028-6020

Yang, L., Qin, L. H., Bligh, S. W., Bashall, A., Zhang, C. F., Zhang, M., et al. (2006). A new phenanthrene with a spirolactone from Dendrobium chrysanthum and its anti-inflammatory activities. Bioorg. Med. Chem. 14, 3496-3501. doi: 10.1016/j. bmc.2006.01.004/0968-0896

Yu, Z., He, C., Teixeira Da Silva, J. A., Luo, J., Yang, Z., and Duan, J. (2018). The GDP-mannose transporter gene (DoGMT) from Dendrobium officinale is critical for mannan biosynthesis in plant growth and development. Plant Sci. 277, 43-54. doi: 10.1016/j.plantsci.2018.07.021/1873-2259

Yuan, Y., Yu, M., Jia, Z., Song, X., Liang, Y., and Zhang, J. (2018). Analysis of Dendrobium huoshanense transcriptome unveils putative genes associated with active ingredients synthesis. BMC Genomics 19:978. doi: 10.1186/s12864-0185305-6/1471-2164

Yuan, Y., Zhang, B., Tang, X., Zhang, J., and Lin, J. (2020). Comparative transcriptome analysis of different Dendrobium species reveals active ingredients-related genes and pathways. Int. J. Mol. Sci. 21:861. doi: 10.3390/ ijms21030861

Zhan, X., Liao, X., Luo, X., Zhu, Y., Feng, S., Yu, C., et al. (2018). Comparative metabolomic and proteomic analyses reveal the regulation mechanism underlying MeJA-induced bioactive compound accumulation in Cutleaf groundcherry (Physalis angulata L.) hairy roots. J. Agricul. Food Chem. 66, 6336-6347. doi: 10.1021/acs.jafc.8b02502/0021-8561

Zhang, G. Q., Xu, Q., Bian, C., Tsai, W. C., Yeh, C. M., Liu, K. W., et al. (2016). The Dendrobium catenatum Lindl. genome sequence provides insights into polysaccharide synthase, floral development and adaptive evolution. Sci. Rep. 6:19029. doi: 10.1038/srep19029/2045-2322

Zhang, J., He, C., Wu, K., Teixeira Da Silva, J. A., Zeng, S., Zhang, X., et al. (2016). Transcriptome analysis of Dendrobium officinale and its application to the identification of genes associated with polysaccharide synthesis. Front. Plant Sci. 7:5. doi: 10.3389/fpls.2016.00005/1664-462X
Zhang, J. H. (1999). Studies on the plant hormones produced by 5 species of endophytic fungi isolated from medicinal plants (Orchidacea). Acta Acad. Med. Sin. 21, 460-465.

Zhang, L., Chen, J., and Lv, Y. (2012). Mycenasp, a mycorrhizal fungus of the orchid Dendrobium officinale. Mycol. Prog. 11, 395-401. doi: 10.1007/s11557011-0754-1

Zhang, X. N., Liu, J., Liu, Y., Wang, Y., Abozeid, A., Yu, Z. G., et al. (2018). Metabolomics analysis reveals that ethylene and methyl jasmonate regulate different branch pathways to promote the accumulation of terpenoid indole alkaloids in Catharanthus roseus. J. Nat. Prod. 81, 335-342. doi: 10.1021/acs. jnatprod.7b00782/1520-6025

Zheng, S. G., Hu, Y. D., Zhao, R. X., Yan, S., Zhang, X. Q., Zhao, T. M., et al. (2018). Genome-wide researches and applications on Dendrobium. Planta 248, 769-784. doi: 10.1007/s00425-018-2960-4

Zhou, X. M., Zheng, C. J., Gan, L. S., Chen, G. Y., Zhang, X. P., Song, X. P., et al. (2016). Bioactive phenanthrene and bibenzyl derivatives from the stems of Dendrobium nobile. J. Nat. Prod. 79, 1791-1797. doi: 10.1021/acs.jnatprod. 6b00252/0163-3864

Zhu, W., Yang, B., Komatsu, S., Lu, X., Li, X., and Tian, J. (2015). Binary stress induces an increase in indole alkaloid biosynthesis in Catharanthus roseus. Front. Plant Sci. 6:582. doi: 10.3389/fpls.2015.00582/1664$462 X$

Zhu, X., Zeng, X., Sun, C., and Chen, S. (2014). Biosynthetic pathway of terpenoid indole alkaloids in Catharanthus roseus. Front. Med. 8:285-293. doi: 10.1007/ s11684-014-0350-2/2095-0225

Conflict of Interest: The authors declare that the research was conducted in the absence of any commercial or financial relationships that could be construed as a potential conflict of interest.

Copyright (c) 2020 Wang, Zhao, Cui, Li and Wang. This is an open-access article distributed under the terms of the Creative Commons Attribution License (CC BY). The use, distribution or reproduction in other forums is permitted, provided the original author(s) and the copyright owner(s) are credited and that the original publication in this journal is cited, in accordance with accepted academic practice. No use, distribution or reproduction is permitted which does not comply with these terms. 